\title{
A NOTE ON LOCALLY FINITE GROUP ALGEBRAS
}

\author{
DANIEL R. FARKAS
}

ABSTRACT. We obtain an injectivity condition for group algebras which is equivalent to local finiteness.

1. Introduction. Several authors have studied the effect of various injectivity conditions on group algebras. Connell [1] showed that if the group algebra $F[G]$ is self-injective, then $G$ is locally finite; Renault [4] improved this result by showing that $G$ is, in fact, finite. The following question arises: what weakening of self-injectivity coincides with local finiteness? This note provides one answer.

We will say that a ring $R$ with 1 is principally (right) self-injective if any right $R$-module map from a principal right ideal of $R$ into $R$ can be lifted to all of $R$. Notice that this definition is the usual Baer criterion for self-injectivity if we omit the two occurrences of the word "principal".

If $M$ is a right $R$-module and $S$ is a subset of $R$, then $l_{M}(S)=$ $\{m \in M \mid m s=0 \forall s \in S\}$ is the left annihilator of $S$ in $M$. Left actions give rise to right annihilators. If $R=M$ we say that a left ideal $L$ of $R$ is a left annihilator if $L=l_{R}(S)$ for some subset $S \leq R$; equivalently, $L=$ $l_{R}\left(r_{R}(L)\right)$. We will drop subscripts when the context is clear.

If $G$ is a group and $F$ is a field, then $F\{G\}$ will denote the set of all infinite formal sums $\Sigma f_{g} g$ with $f_{g} \in F$ and $g \in G$. Under pointwise addition $F\{G\}$ becomes a right $F[G]$-module containing $F[G]$.

Finally we can state our result.

Theorem. The following properties are equivalent:

1. $F[G]$ is principally self-injective.

2. $G$ is locally finite.

3. $F\{G\} \cdot a \cap F[G]=F[G] \cdot a \quad \forall a \in F[G]$.

Received by the editors September 24, 1973 and, in revised form, November 30, 1973 and December 18, 1973.

AMS (MOS) subject classifications (1970). Primary 16A26; Secondary 16A52.

Key words and phrases. Group algebra, injective, annihilator ideal, locally finite group. 
4. Every principal left ideal of $F[G]$ is an annihilator.

The equivalence of 2 and 4 is of particular interest. It might be regarded as a first approximation to the following longstanding conjecture: if every element of $F[G]$ is a zero-divisor or invertible then $G$ is locally finite.

\section{A proof. Crucial to all proofs of local finiteness is}

Lemma [3, p. 105]. Let $g_{1}, \ldots, g_{n}$ be a finite number of elements of $G$, and let $H=\left\langle g_{1}, \cdots, g_{n}\right\rangle$ be the subgroup of $G$ they generate. Then $\left\{r \in F[G] \mid\left(g_{i}-1\right) r=0\right.$ for $\left.i=1, \cdots, n\right\}= \begin{cases}0 & \text { if } H \text { is infinite, } \\ \left(\Sigma_{b \in H} b\right) F[G] & \text { if } H \text { is finite. }\end{cases}$

We proceed to the Theorem:

$1 \Rightarrow 2$. It suffices to prove that $\langle H, x\rangle$ is finite whenever $H$ is a finite subgroup of $G$ and $x \in G$. (One can then argue local finiteness by inducting on the number of generators of a finitely generated subgroup of $G_{\text {. }}$ ) Set $s=\Sigma_{h \in H} h$. The Lemma shows either $\langle H, x\rangle$ is finite or $(x-1)_{s} a=0$ $\Rightarrow s a=0 \forall a \in F[G]$. In the latter case the $F[G]$-map $\phi:(x-1) s F[G] \rightarrow$ $F[G]$ given by $((x-1) s a) \phi=s a$ is well defined. By hypothesis $\exists d \in F[G]$ $\ni s=((x-1) s) \phi=d(x-1) s$, i.e. $(1-d(x-1))_{s}=0$. Since both $d(x-1)$ and any annihilator of $s$ are in the augmentation ideal of $F[G], 1$ is in the augmentation ideal, a contradiction. Thus $\langle H, x\rangle$ is finite.

$2 \Rightarrow 3$. Let $\left\{t_{i}\right\}_{i \in I}$ be a left transversal for the finite subgroup $<$ supp $a>$ $=H$ in $G$. If $\left(\sum_{i \in I} t_{i} b_{i}\right) \cdot a \in F[G]$ with $b_{i} \in F[H]$ then $S=\left\{i \in I \mid b_{i} \cdot a \neq 0\right\}$ is finite. Since $H$ is finite, $\Sigma_{i \in S} t_{i} b_{i} \in F[G]$ and $\left(\Sigma_{I} t_{i} b_{i}\right) \cdot a=\left(\Sigma_{s} t_{i} b_{i}\right) \cdot a$.

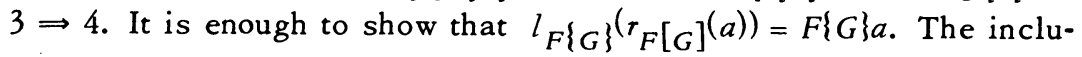
sion " $"$ " is trivial. If $b$ is in the double annihilator then the $F$-linear map $\tau: a F[G] \rightarrow F$ given by $\tau(a r)=\operatorname{tr}(b r)$ is well defined. (Here, $\operatorname{tr}$ of an element in $F\{G\}$ denotes the coefficient of 1.) Lift $\tau$ to an $F$-linear map on $F[G]$. Writing $a=\Sigma_{h} a_{h} h$, a finite sum, we have

$$
\begin{aligned}
b & =\sum_{g \in G} \operatorname{tr}\left(b g^{-1}\right) g=\sum_{g} \tau\left(a g^{-1}\right) g=\sum_{g} \tau\left(\sum_{b} a_{b} b g^{-1}\right) g \\
& =\sum_{g}\left(\sum_{h} a_{h} \tau\left(b g^{-1}\right)\right) g=\sum_{h}\left(\sum_{g} \tau\left(b g^{-1}\right) g b^{-1}\right) a_{h} b=\left(\sum_{\gamma \in G} \tau\left(\gamma^{-1}\right) \gamma\right) a \in F\{G\} a .
\end{aligned}
$$

$4 \Rightarrow 1$. It is easy to see that any ring $R$ is principally right selfinjective iff for each $a \in R, R a=l(r(a))$ [2, Theorem 1]. 


\section{REFERENCES}

1. I. G. Connell, On the group ring, Canad. J. Math. 15 (1963), 650-685. MR $27 \# 3666$.

2. M. Ikeda and T. Nakayama, On some characteristic properties of quasiFrobenius and regular rings, Proc. Amer. Math. Soc. 5 (1954), 15-19. MR 15, 677.

3. D. S. Passman, Infinite group rings, Dekker, New York, 1971.

4. G. Renault, Sur les anneaux de groupes, C. R. Acad. Sci. Paris Sér. A-B 273 (1971), A84-A87. MR $44 \# 5387$.

DEPARTMENT OF MATHEMATICS, BRANDEIS UNIVERSITY, WALTHAM, MASSACHUSETTS 02154 